\title{
Generalized omni-Lie algebras
}

\author{
Chang Sun (D), Liangyun Chen*(D) \\ School of Mathematics and Statistics, Northeast Normal University, Changchun 130024, China
}

\begin{abstract}
We introduce the notion of generalized omni-Lie algebras from omni-Lie algebras constructed by Weinstein. We prove that there is a one-to-one correspondence between Dirac structures of a generalized omni-Lie algebra and Lie structures on its linear space.
\end{abstract}

Mathematics Subject Classification (2010). 17B99, 55U15

Keywords. omni-Lie algebras, generalized omni-Lie algebras, Dirac structures

\section{Introduction}

The notion of omni-Lie algebras was introduced by Weinstein[7], which is the linearization of the Courant bracket. Let $V$ be a linear space, and an omni-Lie algebra is the direct sum space $g l(V) \oplus V$ with a skew-symmetric bracket operation $\llbracket \cdot, \cdot \rrbracket$ and a non-degenerate symmetric bilinear pairing $\langle\cdot, \cdot\rangle$ given by

$$
\llbracket A+x, B+y \rrbracket=[A, B]+\frac{1}{2}(A y-B x),
$$

and

$$
\langle A+x, B+y\rangle=\frac{1}{2}(A y+B x) .
$$

An omni-Lie algebra is not a Lie algebra, but its Dirac structures are Lie algebras. Actually, an omni-Lie algebra is a Lie 2-algebra since Roytenberg and Weinstein proved that every Courant algeboid gives rise to a Lie 2-algebra[5]. Recently, omni-Lie algebras were generalized to omni-Lie superalgebras, omni-Lie color algebras and omni-Lie algebroids $[1,8]$. $\operatorname{In}[2]$, they generalized omni-Lie algebras from a linear space to a linear bundle $E$ in order to characterize all possible Lie algebroid structures on $E$. Dirac structures were also studied from several aspects[2,3,6].

In this paper, we introduce the notion of a generalized omni-Lie algebra, which is the $(\delta, \alpha)$ omni-Lie algebra and discuss special situations when $\delta, \alpha$ are fixed values. Then we study Dirac structures of the generalized omni-Lie algebra in order to characterize all Lie algebra structures on the linear space and prove that there is a one-to-one correspondence between Dirac structures of the generalized omni-Lie algebra $(\Omega, \llbracket \cdot, \cdot \rrbracket,\langle\cdot, \cdot\rangle)$ and Lie algebra structures on subspaces of $V$ if $\delta=\frac{1}{2}$. Moreover, we prove that a generalized omni-Lie algebra is a Leibniz algebra.

\footnotetext{
*Corresponding Author.

Email addresses: sunc015@nenu.edu.cn (C. Sun), chenly640@nenu.edu.cn (L. Chen)

Received: 22.06.2017; Accepted: 20.12.2018
} 


\section{Generalized omni-Lie algebras}

Let $V$ be a linear space over a field $\mathrm{F}$. The set of all linear transformations on $V$ is a Lie algebra denoted by $g l(V)$, given by $[A, B]=A B-B A$, for any $A, B \in g l(V)$.

Definition 2.1. A generalized omni-Lie algebra is the linear space $\Omega=g l(V) \oplus V$ with a skew-symmetric bilinear bracket operation $\llbracket \cdot, \cdot \rrbracket$ and a non-degenerate symmetric bilinear pairing $\langle\cdot, \cdot\rangle$, for any $A, B \in g l(V), x, y \in V$, and $\delta, \alpha \in \mathrm{F}$,

$$
\llbracket A+x, B+y \rrbracket=[A, B]+\delta(A y-B x),
$$

and

$$
\langle A+x, B+y\rangle=\alpha(A y+B x) .
$$

We call $(\Omega, \llbracket \cdot, \cdot \rrbracket,\langle\cdot, \cdot\rangle)$ a generalized omni-Lie algebra.

Proposition 2.2. Let $J$ denote the Jacobiator for the bracket $\llbracket \cdot, \cdot \rrbracket$ of $\Omega$, then for any $e_{1}=A+x, e_{2}=B+y, e_{3}=C+z \in \Omega$,

$$
J\left(e_{1}, e_{2}, e_{3}\right)=\llbracket \llbracket e_{1}, e_{2} \rrbracket, e_{3} \rrbracket+\llbracket \llbracket e_{2}, e_{3} \rrbracket, e_{1} \rrbracket+\llbracket \llbracket e_{3}, e_{1} \rrbracket, e_{2} \rrbracket .
$$

(i) If $\delta=0,1,(\Omega, \llbracket \cdot, \cdot \rrbracket)$ is a Lie algebra.

(ii) If $\alpha=\delta=\frac{1}{2},(\Omega, \llbracket \cdot \cdot, \rrbracket,\langle\cdot, \cdot\rangle)$ is an omni-Lie algebra.

Proof. By a direct calculation, we get

$$
\begin{aligned}
& J\left(e_{1}, e_{2}, e_{3}\right) \\
= & \llbracket \llbracket A+x, B+y \rrbracket, C+z \rrbracket+c . p . \\
= & \llbracket[A, B]+\delta(A y-B x), C+z \rrbracket+c . p . \\
= & {[[A, B], C]+[[B, C], A]+[[C, A], B]+\delta([A, B] z-\delta C(A y-B x)) } \\
& +\delta([B, C] x-\delta A(B z-C y))+\delta([C, A] y-\delta B(C x-A z)) \\
= & {[[A, B], C]+[[B, C], A]+[[C, A], B]+\left(\delta-\delta^{2}\right)([A, B] z+[B, C] x+[C, A] y) } \\
= & \left(\delta-\delta^{2}\right)([A, B] z+[B, C] x+[C, A] y) .
\end{aligned}
$$

If $\delta=0,1, J$ satisfies the Jocabi identity, so $(\Omega, \llbracket \cdot, \cdot \rrbracket)$ is a Lie algebra. Especially if $\delta=1$, $(\Omega, \llbracket \cdot, \rrbracket)$ is a semidirect product of $g l(V)$ and $V$.

Proposition 2.3. Let $J$ denote the Jacobiator for the bracket $\llbracket \cdot, \cdot \rrbracket$ of $\Omega$, for any $e_{1}=$ $A+x, e_{2}=B+y, e_{3}=C+z \in \Omega$, we set

$$
\begin{gathered}
T\left(e_{1}, e_{2}, e_{3}\right)=\left\langle\llbracket e_{1}, e_{2} \rrbracket, e_{3}\right\rangle+\left\langle\llbracket e_{2}, e_{3} \rrbracket, e_{1}\right\rangle+\left\langle\llbracket e_{3}, e_{1} \rrbracket, e_{2}\right\rangle, \\
T^{\prime}\left(e_{1}, e_{2}, e_{3}\right)=\frac{\delta-\delta^{2}}{\alpha+\alpha \delta} T\left(e_{1}, e_{2}, e_{3}\right),
\end{gathered}
$$

then we have

$$
T^{\prime}\left(e_{1}, e_{2}, e_{3}\right)=J\left(e_{1}, e_{2}, e_{3}\right) .
$$

Proof. We have proved that for any $e_{1}=A+x, e_{2}=B+y, e_{3}=C+z \in \Omega$,

$$
J\left(e_{1}, e_{2}, e_{3}\right)=\left(\delta-\delta^{2}\right)([A, B] z+[B, C] x+[C, A] y) .
$$

By Definition 2.1, we get

$$
\begin{aligned}
& T\left(e_{1}, e_{2}, e_{3}\right) \\
= & \left\langle\llbracket e_{1}, e_{2} \rrbracket, e_{3}\right\rangle+c . p . \\
= & \langle[A, B]+\delta(A y-B x), C+z\rangle+c . p . \\
= & \alpha([A, B] z+\delta C(A y-B x))+\alpha([B, C] x+\delta A(B z-C y)) \\
& +\alpha([C, A] y+\delta B(C x-A z)) \\
= & (\alpha+\alpha \delta)([A, B] z+[B, C] x+[C, A] y),
\end{aligned}
$$




$$
T^{\prime}\left(e_{1}, e_{2}, e_{3}\right)=\frac{\delta-\delta^{2}}{\alpha+\alpha \delta} T\left(e_{1}, e_{2}, e_{3}\right)=J\left(e_{1}, e_{2}, e_{3}\right) .
$$

Thus, Eq. (2.3) holds.

Let $\omega$ be a bilinear operation on $V$, and define the adjoint operator $\operatorname{ad}_{\omega}: V \rightarrow g l(V)$ by

$$
\operatorname{ad}_{\omega}(x)(y):=\omega(x, y), \forall x, y \in V,
$$

then the graph of the adjoint operator

$$
\mathscr{F}_{\omega}=\left\{\operatorname{ad}_{\omega}(x)+x, \forall x \in V\right\}
$$

is a subspace of $\Omega$. $\mathscr{F}_{\omega}^{\perp}$ denote the orthogonal complement of $\mathscr{F}_{\omega}$ with respect to the bilinear form (2.2) of $\Omega$.

Proposition 2.4. If $\delta=\frac{1}{2},(V, \omega)$ is a Lie algebra if and only if $\mathscr{F}_{\omega}$ is maximal isotropic, i.e.,

$$
\mathscr{F}_{\omega}=\mathscr{F}_{\omega}^{\perp},
$$

and is closed under the bracket $\llbracket \cdot, \cdot \rrbracket$.

Proof. First, for any $\operatorname{ad}_{\omega}(x)+x, \operatorname{ad}_{\omega}(y)+y \in \mathscr{F}_{\omega}$,

$$
\begin{aligned}
& \left\langle\operatorname{ad}_{\omega}(x)+x, \operatorname{ad}_{\omega}(y)+y\right\rangle \\
= & \alpha\left(\operatorname{ad}_{\omega}(x)(y)+\operatorname{ad}_{\omega}(y)(x)\right) \\
= & \alpha(\omega(x, y)+\omega(y, x)),
\end{aligned}
$$

which means that $\omega(\cdot, \cdot)$ is skew-symmetric if and only if its graph is isotropic and $\mathscr{F}_{\omega}=$ $\mathscr{F} \stackrel{\perp}{\omega}$. Then if $\delta=\frac{1}{2}$ and $\omega(\cdot, \cdot)$ is skew-symmetric, let us check

$$
\begin{aligned}
& \llbracket \operatorname{ad}_{\omega}(x)+x, \operatorname{ad}_{\omega}(y)+y \rrbracket \\
= & {\left[\operatorname{ad}_{\omega}(x), \operatorname{ad}_{\omega}(y)\right]+\frac{1}{2}\left(\operatorname{ad}_{\omega}(x)(y)-\operatorname{ad}_{\omega}(y)(x)\right) } \\
= & {\left[\operatorname{ad}_{\omega}(x), \operatorname{ad}_{\omega}(y)\right]+\frac{1}{2}(\omega(x, y)-\omega(y, x)) } \\
= & {\left[\operatorname{ad}_{\omega}(x), \operatorname{ad}_{\omega}(y)\right]+\omega(x, y) . }
\end{aligned}
$$

Hence, the bracket is closed if and only if

$$
\left[\operatorname{ad}_{\omega}(x), \operatorname{ad}_{\omega}(y)\right]=\operatorname{ad}_{\omega}(\omega(x, y)),
$$

it follows that for any $z \in V$,

$$
\begin{aligned}
& {\left[\operatorname{ad}_{\omega}(x), \operatorname{ad}_{\omega}(y)\right](z)-\operatorname{ad}_{\omega}(\omega(x, y))(z) } \\
= & \operatorname{ad}_{\omega}(x) \operatorname{ad}_{\omega}(y)(z)-\operatorname{ad}_{\omega}(y) \operatorname{ad}_{\omega}(x)(z)-\operatorname{ad}_{\omega}(\omega(x, y))(z) \\
= & \operatorname{ad}_{\omega}(x) \omega(y, z)-\operatorname{ad}_{\omega}(y) \omega(x, z)-\omega(\omega(x, y), z) \\
= & \omega(x, \omega(y, z))-\omega(y, \omega(x, z))-\omega(\omega(x, y), z),
\end{aligned}
$$

it is clear that the bracket is closed if and only if the Jacobi identity of $\omega(\cdot, \cdot)$ on $V$ is satisfied. Thus, the proof is completed.

Definition 2.5. Let $L$ be a maximal isotropic subspace of $\Omega=g l(V) \oplus V$ and closed under the bracket $\llbracket \cdot, \cdot \rrbracket$, then we call $L$ a Dirac structure of the generalized omni-Lie algebra $(\Omega, \llbracket \cdot, \cdot \rrbracket,\langle\cdot, \cdot\rangle)$.

Remark 2.6. By Proposition 2.3, for a Dirac structure $L$, we can get

$$
T^{\prime}\left(e_{1}, e_{2}, e_{3}\right)=J\left(e_{1}, e_{2}, e_{3}\right)=0, \forall e_{i} \in L, i=1,2,3 .
$$

then a Dirac structure $(L, \llbracket \cdot, \cdot \rrbracket)$ is a Lie algebra. 
According to the Definition 2.5, we can rewrite Proposition 2.4 that " if $\delta=\frac{1}{2},(V, \omega)$ is a Lie algebra if and only if $\mathscr{F}_{\omega}$ is a Dirac structure of the generalized omni-Lie algebra $(\Omega, \llbracket \cdot, \cdot \rrbracket,\langle\cdot, \cdot\rangle) . "$

Then we want to know the concrete form of Dirac structures, for a maximal isotropic subspace $L$, let $D=L \cap g l(V)$, define $D^{0}$ to be the kernel of $D$,

$$
\begin{gathered}
D^{0}:=\{x \in V \mid X(x)=0, \forall X \in D\} \subseteq V, \\
\left(D^{0}\right)^{0}:=\left\{X \in g l(V) \mid X(x)=0, \forall x \in D^{0}\right\} \subseteq g l(V)=D .
\end{gathered}
$$

Lemma 2.7. A subspace $L$ is maximal isotropic if and only if

$$
L=D \oplus \mathscr{F}_{\pi \mid D_{0}}=\left\{X+\pi(x)+x \mid \forall X \in D, x \in D^{0}\right\},
$$

where $\pi: V \rightarrow g l(V)$ is a skew-symmetric map.

Proof. First, suppose that $L$ is given by (2.4), for any $X+\pi(x)+x, Y+\pi(y)+y \in L$,

$$
\begin{aligned}
& \langle X+\pi(x)+x, Y+\pi(y)+y\rangle \\
= & \alpha(X(y)+\pi(x)(y)+Y(x)+\pi(y)(x)) \\
= & \alpha(\pi(x)(y)+\pi(y)(x)) \\
= & 0 .
\end{aligned}
$$

Thus, $L$ is isotropic, then we prove that $L$ is maximal isotropic. For all $B+z \in L^{\perp}$, we have

So $z \in D^{0}$,

$$
0=\langle X, B+z\rangle=\alpha X(z), \forall X \in D
$$

$$
\begin{aligned}
& 0=\langle X+\pi(x)+x, B+z\rangle \\
& =\alpha(X(z)+\pi(x)(z)+B(x)) \\
& =\alpha(B-\pi(z))(x), \forall X+\pi(x)+x \in L,
\end{aligned}
$$

let $Z:=B-\pi(z) \in D$,

$$
B+z=Z+\pi(z)+z \in L^{\perp}=L,
$$

therefore, $L$ is maximal isotropic. The converse part is straightforward, so we omit the details.

Lemma 2.8. Let $(D, \pi)$ be given above for a maximal isotropic subspace $L \subset \Omega$. Then $L$ is a Dirac structure if and only if the following conditions are satisfied:

(i) $D$ is a subalgebra of $g l(V)$;

(ii) $\pi(\pi(x, y))-[\pi(x), \pi(y)] \in D, \forall x, y \in D^{0}$;

(iii) $\pi(x, y) \in D^{0}, \forall x, y \in D^{0}$.

Such a pair $(D, \pi)$ is called a characteristic pair of a Dirac structure $L$.

Proof. By Definition 2.5, $L$ is a Dirac structure if and only if $L$ is closed with respect to the bracket (2.1). First, for any $X+\pi(x)+x, Y+\pi(y)+y \in L$, by straightforward calculation, we get

$$
\begin{aligned}
& \llbracket X+\pi(x)+x, Y+\pi(y)+y \rrbracket \\
= & {[X+\pi(x), Y+\pi(y)]+\delta(\pi(x)(y)-\pi(y)(x)) } \\
= & {[X, Y]+[X, \pi(y)]+[\pi(x), Y]+[\pi(x), \pi(y)]+2 \delta \pi(x, y) . }
\end{aligned}
$$

If $\delta=\frac{1}{2}, L$ is closed under the bracket (2.1) if and only if $\pi(x, y) \in D^{0}, \pi(\pi(x, y))-$ $[\pi(x), \pi(y)] \in D, \forall x, y \in D^{0}$. Moreover for any $X, Y \in D, x, y, z \in D^{0}$, we have

$$
\begin{gathered}
{[X, Y](z)=X Y(z)-Y X(z)=0,} \\
{[X, \pi(y)](z)=X \pi(y)(z)-\pi(y) X(z)=0,} \\
{[\pi(x), Y](z)=\pi(x) Y(z)-Y \pi(x)(z)=0,}
\end{gathered}
$$


so $[X, Y],[X, \pi(y)],[\pi(x), Y] \in D$, that is to say, $D$ is a subalgebra of $g l(V)$.

Theorem 2.9. There is a one-to-one correspondence between Dirac structures of the generalized omni-Lie algebra $(\Omega, \llbracket \cdot, \cdot \rrbracket,\langle\cdot, \cdot\rangle)$ and Lie algebra structures on subspaces of $V$ if $\delta=\frac{1}{2}$.

Proof. First, by Lemmas 2.7, 2.8, if $L$ is a Dirac structure, then $L=D \oplus \mathscr{F}_{\pi \mid D_{0}}$ and satisfies three conditions in Lemma 2.8. Define operation $[\cdot, \cdot \cdot]_{D^{0}}$ on $D^{0} \subseteq V$ by

$$
[x, y]_{D^{0}}:=\pi(x, y) \in D^{0}, \forall x, y \in D^{0},
$$

$[\cdot, \cdot]_{D^{0}}$ is a skew-symmetric operation because $\pi$ is a skew-symmetric map. Then, we check the Jacobi identity, for any $x, y, z \in D^{0}$,

$$
\begin{aligned}
& {\left[[x, y]_{D^{0}}, z\right]_{D^{0}} } \\
= & \pi\left([x, y]_{D^{0}}\right)(z) \\
= & \pi(\pi(x, y))(z) \\
= & {[\pi(x), \pi(y)](z) } \\
= & \pi(x) \pi(y)(z)-\pi(y) \pi(x)(z) \\
= & {\left[x,[y, z]_{D^{0}}\right]_{D^{0}}-\left[y,[x, z]_{D^{0}}\right]_{D^{0}} . }
\end{aligned}
$$

Thus, $\left(D^{0},[\cdot, \cdot]_{D^{0}}\right)$ is a Lie algebra.

Conversely, $W$ is a subspace of $V$, for any Lie algebra $\left(W,[\cdot, \cdot]_{W}\right)$, and define $D$ by

$$
\begin{gathered}
D:=W^{0}=\{X \in g l(V) \mid X(x)=0, \forall x \in W\}, \\
D^{0}=\left(W^{0}\right)^{0}=W .
\end{gathered}
$$

Let ad $: W \rightarrow g l(W)$ represents the limitation of $\pi: V \rightarrow g l(V)$ on $W$,

$$
\operatorname{ad}_{x}(y)=[x, y]_{W},
$$

then we get a maximal isotropic subspace

$$
L=D \oplus \mathscr{F}_{\pi \mid W} .
$$

Next is to prove that $L$ is closed under the bracket $\llbracket \cdot, \cdot \rrbracket$, if $\delta=\frac{1}{2}$, for $X+\operatorname{ad}_{x}+x, Y+$ $\operatorname{ad}_{y}+y \in L$,

$$
\begin{aligned}
& \llbracket X+\operatorname{ad}_{x}+x, Y+\operatorname{ad}_{y}+y \rrbracket \\
= & {\left[X+\operatorname{ad}_{x}, Y+\operatorname{ad}_{y}\right]+\frac{1}{2}\left(\left(X+\operatorname{ad}_{x}\right)(y)-\left(Y+\operatorname{ad}_{y}\right)(x)\right) } \\
= & {[X, Y]+\left[X, \operatorname{ad}_{y}\right]+\left[\operatorname{ad}_{x}, Y\right]+\left[\operatorname{ad}_{x}, \operatorname{ad}_{y}\right]+\frac{1}{2}\left(\operatorname{ad}_{x}(y)-\operatorname{ad}_{y}(x)\right) } \\
= & {[X, Y]+\left[X, \operatorname{ad}_{y}\right]+\left[\operatorname{ad}_{x}, Y\right]+\left[\operatorname{ad}_{x}, \operatorname{ad}_{y}\right]+[x, y]_{W} . }
\end{aligned}
$$

For any $X, Y \in D$ and $x, y \in W$,

$$
[X, Y](x)=X Y(x)-Y X(x)=0,
$$

which means $[X, Y] \in D, D$ is a subalgebra of $g l(V)$.

$$
\begin{aligned}
& {\left[X, \operatorname{ad}_{x}\right](y)=X\left([x, y]_{W}\right)-[x, X(y)]=0,} \\
& {\left[\operatorname{ad}_{x}, Y\right](y)=[x, Y(y)]-Y\left([x, y]_{W}\right)=0,}
\end{aligned}
$$

so $\left[X, \operatorname{ad}_{y}\right],\left[\operatorname{ad}_{x}, Y\right] \in D$.

Since $[\cdot, \cdot]_{W}$ satisfies the Jacobi identity, we obtain

$$
\begin{gathered}
{\left[\operatorname{ad}_{x}, \operatorname{ad}_{y}\right]=\operatorname{ad}_{[x, y]_{W}},} \\
\llbracket X+\pi(x)+x, Y+\pi(y)+y \rrbracket \in D \oplus \mathscr{F}_{\pi \mid W} .
\end{gathered}
$$

Thus, $L$ is a Dirac structure. 
Let $\Lambda$ denotes the family of all Lie structures on the subspaces of $V$, and $\Gamma$ denotes the family of all Dirac structures of the generalized omni-Lie algebra $\Omega$, then according to Theorem 2.9, there exists a bijective

$$
\Psi: \Lambda \rightarrow \Gamma
$$

and an embedding

$$
\varphi_{W}: W \rightarrow L, \forall W \in \Lambda, L \in \Gamma .
$$

Definition 2.10. [4] Let $L$ be a linear space over a field $F$ together with a bilinear operation $\circ: L \times L \rightarrow L$ satisfying

$$
((x \circ y) \circ z)=(x \circ(y \circ z))-(y \circ(x \circ z)), \forall x, y, z \in L,
$$

then we call $(L, \circ)$ a Leibniz algebra.

We define another bilinear operation "o" on $\Omega=g l(V) \oplus V$ by

$$
(A+x) \circ(B+y)=[A, B]+\delta A y, \forall A+x, B+y \in \Omega, \delta \in \mathrm{F} .
$$

Proposition 2.11. $(\Omega, \circ)$ is a Leibniz algebra.

Proof. We check if the Leibniz identity is satisfied, for any $e_{1}=A+x, e_{2}=B+y, e_{3}=$ $C+z \in \Omega$,

$$
\begin{aligned}
& \left(e_{1} \circ e_{2}\right) \circ e_{3}-e_{1} \circ\left(e_{2} \circ e_{3}\right)+e_{2} \circ\left(e_{1} \circ e_{3}\right) \\
= & ([A, B]+\delta A y) \circ(C+z)-(A+x) \circ([B, C]+\delta B z)+(B+y) \circ([A, C]+\delta A z) \\
= & {[[A, B], C]-[A,[B, C]]+[B,[A, C]]+\delta(A B z-B A z)-\delta A B z+\delta B A z } \\
= & 0 .
\end{aligned}
$$

By Definition 2.10, it holds.

Proposition 2.12. Let $V$ be a Lie algebra. $D$ is a derivation of $V$ that satisfies

$$
D[x, y]=[D x, y]+[x, D y], \forall x, y \in V
$$

if and only if $\mathscr{F}_{\omega}$ is an invariant subspace of $D$ under the operation "o" if $\delta=1$, i.e.,

$$
D \circ \mathscr{F}_{\omega} \subseteq \mathscr{F}_{\omega} .
$$

Proof. If $\delta=1$, for $\operatorname{ad}_{\omega}(x)+x \in \mathscr{F}_{\omega}, y \in V$,

$$
D \circ\left(\operatorname{ad}_{\omega}(x)+x\right)=\left[D, \operatorname{ad}_{\omega}(x)\right]+D x .
$$

The right side belongs to $\mathscr{F}_{\omega}$ if and only if

$$
\left[D, \operatorname{ad}_{\omega}(x)\right]=\operatorname{ad}_{\omega}(D x),
$$

for convenience, we denote $\omega(x, y):=[x, y]$,

$$
\begin{aligned}
& {\left[D, \operatorname{ad}_{\omega}(x)\right](y)-\operatorname{ad}_{\omega}(D x)(y) } \\
= & D \operatorname{ad}_{\omega}(x)(y)-\operatorname{ad}_{\omega}(x) D(y)-\operatorname{ad}_{\omega}(D x)(y) \\
= & D[x, y]-[x, D y]-[D x, y] .
\end{aligned}
$$

Thus, $D$ is a derivation of $V$ if and only if $D \circ \mathscr{F}_{\omega} \subseteq \mathscr{F}_{\omega}$.

Acknowledgment. This work was supported by NNSF of China (No. 11771069), NSF of Jilin province (No. 20170101048JC) and the project of Jilin province Department of Education (No. JJKH20180005K). 


\section{References}

[1] Z. Chen and Z. Liu, Omni-Lie algeboids, J. Geom. Phys. 60 (5), 799-808, 2010.

[2] Z. Chen, Z. Liu and Y. Sheng, Dirac structures of omni-Lie algeboids, Int. J. Math. 22 (8), 1163-1185, 2008.

[3] Z. Liu, Some remarks on Dirac structures and Possion reductions, Poisson Geometry Banach Center Publ. 51, 165-173, 2000.

[4] J. Loday, Une version non commutative des algèbres de Lie: les algèbres de Leibniz, Enseign. Math. 39 (2), 269-293, 1993.

[5] D. Roytenberg and A. Weinstein, Courant algeboids and strongly homotopy Lie algebras, Lett. Math. Phys. 46 (1), 81-93, 1998.

[6] Y. Sheng, Z. Liu and C. Zhu, Omni-Lie 2-algebras and their Dirac structures, J. Geom. Phys. 61 (2), 560-575, 2010.

[7] A. Weinstein, Omni-Lie algebras, RIMS Kôkyûroku, 1176, 95-102, 2000.

[8] T. Zhang and Z. Liu, Omni-Lie superalgebras and Lie 2-superalgebras, Front Math. China, 9 (5), 1195-1210, 2014. 